\title{
Correction to: Identification and Other Probabilistic Models
}

\section{Correction to:}

R. Ahlswede, Identification and Other Probabilistic Models, Foundations in Signal Processing, Communications and Networking 16, https://doi.org/10.1007/978-3-030-65072-8

Due to an unfortunate oversight incorrect information was captured online regarding the authorship of chapters "Testing of Hypotheses and Identification" and "New Results in Identification Theory".

This has been updated online.

\footnotetext{
The updated online version of this chapters can be found at https://doi.org/10.1007/978-3-030-65072-8_23 https://doi.org/10.1007/978-3-030-65072-8_27 